\title{
Suprapatellar swelling and knee instability
}

\author{
Matthieu Ehlinger • Philippe Adam • \\ Guillaume Bierry • Jean Claude Dosch • \\ Gilbert Taglang $\cdot$ Francois Bonnomet
}

Published online: 16 March 2010

(C) ISS 2010

\section{Diagnosis}

Intra-articular lipoma causing patellofemoral instability and subluxation.

\section{Discussion}

Intra-articular knee masses are rare, with an incidence of only 33 out of 2,200 knee arthroscopies described in the literature [1]. Lipomas are common benign tumours, but an intra-articular localisation is exceptional. Two types of lipomatous entities can be found: solitary lipoma and lipoma arborescens. They differ in pathogenesis and gross appearance, although their microscopic features are similar.

The case presentation can be found at doi:10.1007/s00256-010-0877-5.

M. Ehlinger $(\bowtie) \cdot$ P. Adam $\cdot$ G. Taglang $\cdot$ F. Bonnomet

Service de Chirurgie Orthopédique et Traumatologie,

CHU Hautepierre, Hôpitaux Universitaires de Strasbourg,

Hôpital de Hautepierre,

1 Avenue Molière,

67098 Strasbourg, France

e-mail: matthieu.ehlinger@chru-strasbourg.fr

P. Adam

e-mail: philippe.adam@chru-strasbourg.fr

G. Taglang

e-mail: gilbert.taglang@chru-strasbourg.fr

F. Bonnomet

e-mail: francois.bonnomet@chru-starsbourg.fr

G. Bierry $\cdot$ J. C. Dosch

Service de Radiologie, Hôpitaux Universitaires de Strasbourg,

Hôpital de Hautepierre,

Strasbourg, France

G. Bierry

e-mail: guillaume.bierry@chru-starsbourg.fr

J. C. Dosch

e-mail: jeanclaude.dosch@chru-strasbourg.fr
Lipoma arborescens, the more frequent of the two intraarticular tumours, is usually considered to be a secondary lesion, occurring in the context of either inflammatory or degenerative joint disease, or after trauma [2-4]. Microscopically, lipoma arborescens is manifested as a diffuse hyperplasic villous proliferation of the synovium in response to chronic irritation. Magnetic resonance imaging (MRI) examination is pathognomonic, showing multinodular fatty tissue infiltrating and protruding into the joint. The tissue shows contrast enhancement, and an effusion is often present.

Solitary intra-articular lipoma is not associated with preexisting pathology, and no causative factors can be identified. Macroscopically, it is a soft yellow solitary mass, round or ovoid in shape, uni- or poly-lobed, and finely encapsulated. It is usually small and often pediculated. It is thought to develop from the fat of the suprapatellar or retropatellar regions. On MRI the fatty signal shows increased intensity in $\mathrm{T} 1$ sequences and decreased intensity in all the sequences with saturation of the fat. There is no enhancement after injection of contrast medium.

Seventeen cases of intra-articular lipoma of the knee were found in the international literature. This lesion is a rare cause of pain or internal articular dysfunction of the knee. Clinical presentation may vary from a simple palpable mass to a more complex association of pain, swelling and internal derangement. There is a relationship between anatomical localisation and clinical presentation. Tumours of the suprapatellar region usually present as a simple palpable mass. However, localisation within Hoffa's fat pad or between the femur and tibia may have more varied manifestations, with pain, reduced range of motion and joint locking.

Our case was an unusually large tumoral mass with a peculiar presentation. To our knowledge, it is the first example of patellofemoral instability with patellar subluxation due to intra-articular lipoma, although there has been one previously reported case with perturbation of patellofemoral tracking [5]. 
Treatment is surgical. The type of surgery is adapted to the size of the lipoma. As these tumours are often small, resection is most often done arthroscopically. Arthrotomy is preferred for lipomas exceeding $4 \mathrm{~cm}$ in size [6].

\section{References}

1. Ozalay M, Tandogan RN, Akpinar S, et al. Arthroscopic treatment of solitary benign intra-articular lesions of the knee that cause mechanical symptoms. Arthroscopy. 2005;21:12-8.
2. Kloen P, Keel SB, Chandler HP, Geiger RH, Zarins B, Rosenberg AE. Lipoma arborescens of the knee. J Bone J Surg Br. 1998;80:298-301.

3. Sola JB, Wright RW. Arthroscopic treatment for lipoma arborescens of the knee. J Bone J Surg Am. 1998;80:99-103.

4. Ikushima K, Ueda T, Kudawara I, Yoshikawa H. Lipoma arborescens of the knee as a possible cause of osteoarthrosis. Orthopedics. 2001;24:603-5.

5. Yilmaz E, Karakurt L, Yildirim H, Ozercan R. Intra-articular lipoma causing snapping in the patellofemoral joint. Saudi Med J. 2007;28:955-8.

6. Marui T, Yamamoto T, Kimura T, et al. A true intra-articular lipoma of the knee in a girl. Arthroscopy. 2002;18:E24. 